\title{
Tailoring of magnetic hyperthermia treatment using superparamagnetic iron oxide nanoparticles
}

\begin{abstract}
Despite the latest advancement in diagnosis and treatments, cancer is among the top three causes of death in modern society. Engineered nanoparticles have the potential to revolutionize the diagnosis and treatment of cancer. Superparamagnetic iron oxide nanoparticles (SPIONs) have acted as the most promising candidate in magnetic hyperthermia treatment of cancer. Use of iron oxide super paramagnetic nanoparticle will lead to increased intracellular concentration of drugs. Unique properties of iron oxide nanoparticles have helped to overcome the limitations of cancer therapy such as lethal dosage of radiation and harmful side effects. In this review, we have highlighted the recent advances in treatment of magnetic hyperthermia using SPIONs
\end{abstract}

Keywords: magnetic hyperthermia, iron oxide nanoparticles, superparamagnetic, neel relaxation, brownian relaxation, alternating magnetic field, specific absorption rate, thermal loss
Volume 2 Issue | - 2017

\author{
Jay Pala, Hetal Mehta, Ravi Mandaliya, Meet \\ Moradiya, Chirag R Savaliya, Markna JH \\ Department of Nanotechnology, Gujarat Technological \\ University, India
}

Correspondence: Jay Pala, Department of Nanotechnology, Gujarat Technological University, Rajkot-36005, India, Tel (028I) 2783394, (028I) 2783486, Fax (028I) 2783487, Email jaysukh28@gmail.com

Received: July 29, 2017 | Published: October 10, 2017

\section{Introduction}

Cancer is one of the fatal diseases leading to 8.8 million deaths across the world in 2015. In this desperate scenario, nanotechnology has a leading edge for the cancer treatment. Using iron oxide nanoparticles in magnetic hyperthermia leads to advanced and efficient methodology in the form of treatment that nearly assures $100 \%$ results for cancer curing. Magnetic hyperthermia is a treatment that destroys cancerous tumor cells with the help of magnetic nanoparticles (MNPs) as they generate heat in the presence of an alternating magnetic field. ${ }^{1}$ MNPs like iron oxide exhibits superparamagnetic property as they easily get magnetized and rapidly demagnetized on applying and removal of the external magnetic field without any hysteresis losses. Iron oxide nanoparticles especially magnetite and maghemite have the set of properties that are very well suited for the magnetic hyperthermia treatment of cancerous tumor cells.

\section{Iron oxide nanoparticles for magnetic hyperthermia}

From a large variety of magnetic nanoparticles, magnetite $\left(\mathrm{Fe}_{3} \mathrm{O}_{4}\right)$ and maghemite $(\gamma-\mathrm{Fe} 2 \mathrm{o} 3)$ are mostly used due to their good biocompatibility, low toxicity, surface functionalization and ease synthesis. ${ }^{2}$ These properties make them applicable for biomedical applications like magnetic hyperthermia and targeted drug delivery. Maghemite and magnetite nanoparticles have been easily synthesized previously with the help of various preparation techniques such as sol-gel, co-precipitation or hydrothermal. O M Lemine et al. ${ }^{2}$ used a modified sol-gel method to prepare $14 \mathrm{~nm}$ diameter iron oxide $\gamma-\mathrm{Fe}_{2} \mathrm{O}_{3}$ nanoparticles under supercritical conditions of ethyl alcohol. Whereas, Michael Levy et al. ${ }^{3}$ used alkaline co-precipitation with an additional step of a hydrothermal method to prepare $\gamma-\mathrm{Fe}_{2} \mathrm{O}_{3}$ (maghemite) NPs. ${ }^{4}$ For additional biocompatibility issue or specific hydrophilic properties most of the time a polymer layer like polyethylene glycol (PEG) is coated on it. Y Lv et al. ${ }^{5}$ prepared magnetite nanoparticles of different sizes using thermal decomposition technique. ${ }^{6}$ In magnetic hyperthermia, the SPIONs are injected into the body intravenously and driven to the cancerous tumor cells with the help of external magnetic field or the specific agents that can be capped on the particles find its way to the tumor itself. The SPIONs group together at the tumor site and after that alternating magnetic field (AMF) is applied. Two phenomena, Neel relaxation (internal) and Brownian relaxation (external) generate heat on an application of an alternating magnetic field. Both internal and external sources of 'friction' lead to a phase lag between the applied magnetic field and the direction of the particle's magnetic moments, thereby generating thermal losses. This thermal loss increases the temperature of tumor cell and ultimately kills it. (Figure 1) represents the procedure for destruction of tumor cells with magnetic hyperthermia performed by SPIONs. SPIONs generate heat only around the tumor site and not all over the body, which is its one of the biggest advantage. Once injected, SPIONs can perform hyperthermia multiple times.

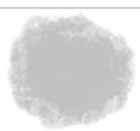

(a)

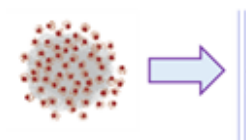

(c)

Cancerous tumor cells

Iron oxide Nanoparticles with polymer coating

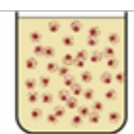

(b)

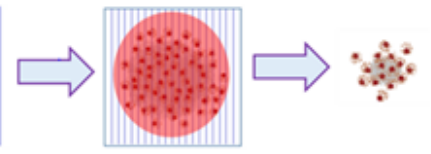

Alternating Magnetic Field (AMF) Heat generation around SPIONs due to AMF
Figure I The steps of magnetic hyperthermia treatment to destroy tumor cell. (A) Cancerous tumor cells, (B) Colloidal system of polymer coated SPIONs, (C) SPIONs gathered around and dispersed in tumor, (D) Alternating magnetic field (AMF) is applied, (E) heat generation due to internal and external friction of SPIONs that destroy tumor cells, (F) Reduced tumor size after application of AFM.

In magnetic hyperthermia factors like nanoparticle diameter, nanoparticle anisotropy, field amplitude and magnetic field frequency affect it greatly. In the size range of $15-25 \mathrm{~nm}$, magnetite and maghemite nanoparticles show highest specific absorption rate (SAR) values. Increasing magnetic field intensity and magnetic field frequency increases the heat rates and SAR values. ${ }^{7}$ Higher SAR values of SPIONs are preferred for magnetic hyperthermia. Andrei Avram et al. performed an experiment in which he applied an AMF of 
$120 \mathrm{kHz}$ and 45 Oe which results in heating of PEG coated maghemite, leading to rise in the temperature of the melanoma cells. OM Lemine et al. $^{2}$ in his article stated that as the field frequency increases from 110 to $523 \mathrm{kHz}$ the temperature of colloids increase significantly. ${ }^{3,5}$

\section{Conclusion}

Advancement of Nanotechnology in the field of cancer treatment has increased tremendously. SPIONs exhibit properties like good biocompatibility, low toxicity, surface functionalization and high specific absorption rate (SAR) value which make them the most promising candidate for magnetic hyperthermia. Optimization in nanoparticle size, particle distribution, and frequency of alternating magnetic field can lead to increase in efficiency of magnetic hyperthermia treatment. High cost, complicated and lethal side effects of at present available cancer treatments can be replaced by SPIONs magnetic hyperthermia.

\section{Acknowledgements}

None.

\section{Conflict of interest}

The author declares no conflict of interest.

\section{References}

1. Salunkhe AB, Khot VM, Pawar SH. Magnetic hyperthermia with magnetic nanoparticles: a status review. Curr Top Med Chem. 2014;14(5):572-594.

2. Markna JH, Raval CS, Shah NA. Current aspects of nanomedicine for cancer. Research \& Reviews: Journal of Oncology and Hematology. 2014;3(1):1-4.

3. Lemine OM, Omri K, El Mir L, et al. $\mathrm{Fe}_{2} \mathrm{O}_{3}$ nanoparticles for magnetic hyperthermia applications. MRS Online Proceedings Library Archive. 2015;1779:7-13

4. Lévy M, Wilhelm C, Siaugue JM, et al. Magnetically induced hyperthermia: size-dependent heating power of $\gamma-\mathrm{Fe}_{2} \mathrm{O}_{3}$ nanoparticles. Journal of Physics: Condensed Matter. 2008;20(20):204133.

5. Avram A, Radoi A, Schiopu V, et al. Synthesis and characterization of $\gamma-\mathrm{Fe}_{2} \mathrm{O}_{3}$ nanoparticles for applications in magnetic hyperthermia. Synthesis. 2011;10(P151):1.

6. Lv Y, Yang Y, Fang J, et al. Size dependent magnetic hyperthermia of octahedral $\mathrm{Fe}_{3} \mathrm{O}_{4}$ nanoparticles. RSC Advances. 2015;5(94):7676476771.

7. Shah RR, Davis TP, Glover AL, et al. Impact of magnetic field parameters and iron oxide nanoparticle properties on heat generation for use in magnetic hyperthermia. J Magn Magn Mater. 2015;387:96-106. 